\title{
Flame Propagation in the Channel and Flammability Limits
}

\author{
G. M. MAKHVILADZE, V. I. MELIKHOV and V. A. RABINKOV \\ Institute for Problems in Mechanics of the USSR Academy of Sciences \\ Prosp. Vernadskogo 101, Moscow 117526, USSR
}

\section{ABSTRACT}

Using full non-stationary two dimensional equations for the gas combustion, the problem of flame propagation in a planar channel with cold side walls is considered. The processes of flame quenching are studied comprehensively. The values of critical peclet numbers are found numerically and analytically, depending on the value of one dimensionless parameter, which characterizes the combustion heat and the composition of the fresh mixture. It is shown that the critical parameters, obtained in the numerical calculations are in satisfactory agreement with the experimental data. The mechanism of the flame propagation and its structure without quenching is considered.

KEYWORDS: flame propagation, flammability limits, flame extinction.

\section{INTRODUCTION}

Two-dimensional flame propagation in a closed planar channel was investigated numerically in [1]. To simplify the problem the side walls were considered as adiabatic. In reality heat losses to the side walls take place. The heat losses into cold walls give rise to a number of new effects, the main of wich is flammability limitation: under definite critical conditions the flame loses ability to propagate through the fresh mixture. Because of the importance of critical phenomena for the understanding of fundamental flame properties, as well as for their application, much work has been done on flammability limits and there are extensive experimental results [2].

The main principles of the limit theory were developed in [3]. Considering the burning in the narrow tubes, Ya. B. Zeldovich showed that due to feedback between the normal flame velocity and the value of heat losses from the reaction zone, there is the critical normal velocity which is $\sqrt{e}$ times smaller than the normal velocity of the adiabatic flame. Quenching is the result of the conductive heat losses to the walls in the narrow tubes and of radiation heat losses in the wide ones. In [3] the temperature fall at the flammability limit was also calculated. Later similar results were obtained in [4]. 
In this study the flame propagation in a planar closed channel with cold side walls is studied by numerical integration of non-steady two-dimensional equations for the viscous compressible gas. The quenching process is investigated in detail. Critical Peclet numbers are found numerically and analytically. The features of flame propagation in the absence of quenching are considered.

\section{FORMULATION}

Let us consider an immovable combustible mixture in a planar channel with rectangular cross-section $0 \leq \mathrm{X} \leq \mathrm{H}_{\mathrm{O}},-\mathrm{L} / 2 \leq \mathrm{Y} \leq \mathrm{L} / 2$ (Ho, L-the length and the width of the channel, the coordinate system is shown in Fig.1) and solid impenetrable boundaries. In the initial stages the mixture is ignited by hot burning products, filling a small domain near by the center of the left end channel wall. A one-stage exothermic chemical reaction of the first order of limited component and the Arrhenius law for reaction rate is assumed. Thermal properties of the burnt and unburnt gases are considered to be the same. The end channel walls are adiabatic and the side ones have the initial temperature $\mathrm{T}_{0}$ of the cold mixture.

Motion of the reacting mixture is described by the system of two-dimensional unsteady equations. Dimensionless variables are considered as follows. The channel width L; velocity of the flame propagation from the wall $\mathrm{U}_{\mathrm{b}}$ (as defined by Zeldovich - Frank-Kamenetsky formula [2]); time $\mathrm{L} / \mathrm{U}_{\mathrm{b}}$, initial temperature and concentration of the limited component in fresh mixture $T_{0}$ and $a_{0}$; initial gas pressure $P_{0}$ is chosen together with the scales of length, velocity, time, temperature, reagent concentration and pressure. The density scale is expressed from the gas state equation: $p_{0}=P_{0} / R T_{0}$, where $R$ is the gas constant.

In dimensionless variables the system of governing equations is written as:

$\frac{\partial p}{\partial t}+\operatorname{div}(\overrightarrow{\rho U})=0$,

$\frac{\partial \vec{U}}{\partial t}+(\vec{U} \cdot \nabla) \vec{U}=-\frac{1}{M^{2} \rho} \cdot \nabla P+\frac{1}{\operatorname{Re} \rho} \cdot\left(\Delta \vec{U}+\frac{1}{3} \cdot \nabla(\overrightarrow{\nabla U})\right)$,

$\frac{\partial \theta}{\partial t}+(\vec{U} \cdot \nabla) \theta=\frac{\gamma}{\rho \operatorname{RePr}} \cdot \Delta \theta-(\gamma-1) \cdot \theta \cdot \operatorname{div} \vec{U}+q \cdot \operatorname{Dm} \cdot a \cdot \Phi(\theta)$,

$\frac{\partial a}{\partial t}+(\vec{U} \cdot \nabla) a=\frac{L e}{\rho \operatorname{RePr}} \cdot \Delta a-D m \cdot a \cdot \Phi(\theta), P=\rho \cdot \theta$, 
where $\Phi(\theta)=\exp \left[E\left(\theta_{a}^{-1}-\theta^{-1}\right)\right], \Delta \equiv \partial^{2} / \partial x^{2}+\partial^{2} / \partial y^{2}$,

$\mathrm{Dm}=\operatorname{Re} \cdot \operatorname{Pr} \cdot \mathrm{E}^{2} \cdot\left(\theta_{\mathrm{a}}-1\right)^{2} / 2 \cdot \theta_{\mathrm{a}}^{5}$

Here $t$ is the time; $P, \rho, \theta, a$ - the pressure, the density, the gas temperature and the reagent concentration; $\vec{U}(u, v)$-the gas velocity. The dimensionless complexes take the form: $M^{2}=U^{2}{ }_{b} / \gamma R T_{0}-$ is the square of the Mach number $\left(\gamma=c_{p} / c_{v}\right), \quad R e=L U_{b} \rho_{o} / \eta, \operatorname{Pr}=c h / \lambda, L e=\rho_{0} c_{p} D / \lambda$ - the Reynolds, Prandt 1 and Lewis numbers $(n, \lambda, D$ - the coefficients of the dynamic viscosity, the conductivity, the diffusion; the values of $\eta, \lambda$ and $\rho D$ are considered to be constant); $E=\tilde{E} / R_{0} T_{0}, q=0 a_{0} / c_{v} T_{0}(\tilde{E}$ and 0 are the activation energy and the heat of the chemical reaction, $R_{0}$ - universal gas constant); $\Theta_{a}=1+q / \gamma$ - the dimensionless adiabatic temperature of combustion, Dm - the Damkohler number, which with the usual way of consideration of dimensionless values is not independent an parameter, but is given by the above formula.

The formulated problem was integrated numerically by finite-difference method [5]. The main calculations vere carried out on uniform grid with the space step $\mathrm{h}=1 / 20$ and the time step, corresponding to the Courant number, which was defined by means of the sound velocity in the hot gas and equal to 4-5. The following values of parameters were used in the calculations: $\quad r=1.4, M^{2}=0.01, \operatorname{Re}=20 \div 300, \operatorname{Pr}=\mathrm{Le}=1, \mathrm{q}=3 \div 7, \mathrm{H}=4, \mathrm{E}=33$.

\section{RESULTS}

For low Reynolds numbers (Peclet number $\mathrm{Pe}=\mathrm{U}_{b} \mathrm{~L} \mathrm{p}_{\mathrm{o}} \mathrm{C}_{\mathrm{p}} / \lambda$ for gases is approximately equal to the Reynolds number inasmuch as $\mathrm{Pr}=1$ ) the flame is not capable of propagating in the channel with the cold side walls because of heat losses. Calculations showed that for given $q$ the flame quenches within a definite range of the Reynolds number values. Quenching is quicker, the lower the Re number.

Flame quenching is shown in Fig.1. The hot region ignites the surrounding mixture. As a result the flame front, that is the narrow zone, where the heat release and the chemical reaction takes place, is formed (Fig.1,a). Near the cold walls the chemical reaction does not take place because of the low gas temperature. Unlike the burning in the adiabatic channel [1] there is no similarity between the temperature and the concentration fields, that may be explained by the difference in boundary conditions for those values at the side walls.

Because of the heat losses to the walls the hot gases are continuously cooled. Chemical reaction heat is not enough to maintain the high temperature in the burning zone. That is why the zone of the intensive chemical reaction gradually moves away from the channel walls, and concentrates in the middle of the channel, where it eventually disappears, - flame quenches (Fig.1,b,c). 
a)

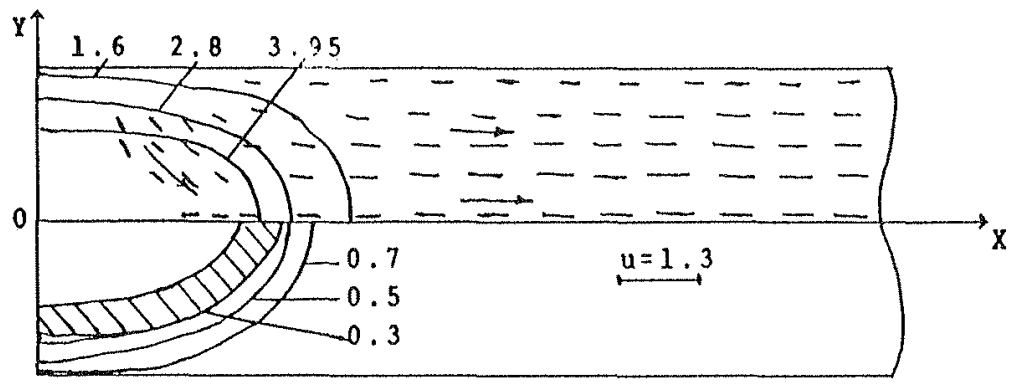

b)

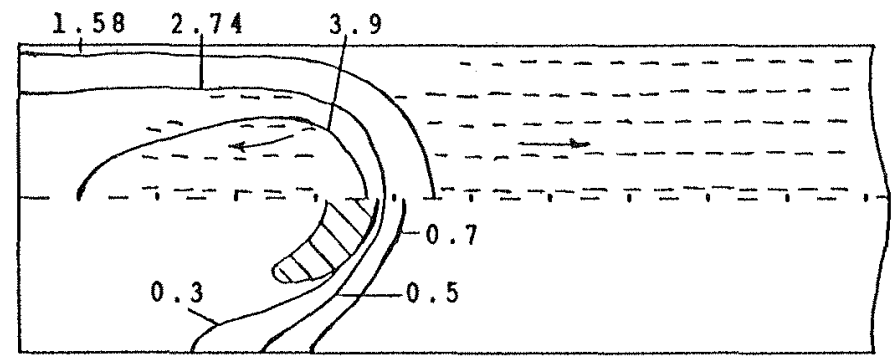

c)

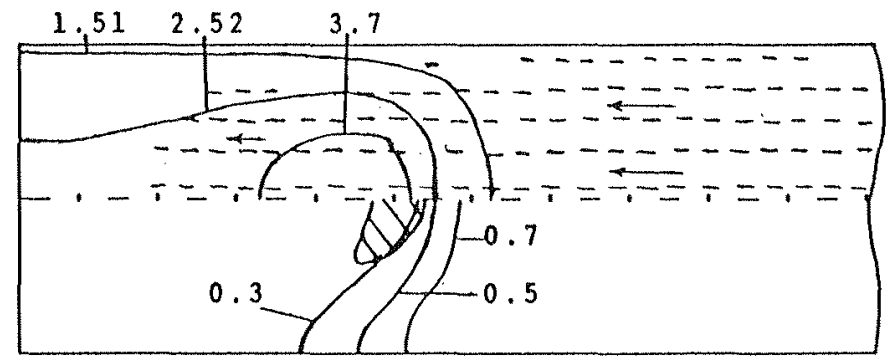

FIGURE 1. The flame quenching, $R e=35, q=5$. a) $t=0.4, b) t=1.2, c) t=2.4$. Shaded region is the burning zone.

The flammability limit is usually characterized by the critical value of the Peclet number Pe*. Numerical modelling carried out here enabled Pe* to be defined as a function of dimensionless parameter $q$, which is proportional to the heat of the reaction and the initial reagent concentration (Fig.2, curves 1,2). In the range of parameters disposed above curve 2 , the flame propagates all over the channel. For the parameters between the curves 1 and 2 only partial burning down takes place. Finally for the system parameters below the curve 1 the mixture cannot be ignited. Let us compare results of numerical two-dimensional solution with the analytical ones, following from the one-dimensional theory. With that end of view, it's necessary to define the critical Peclet number on the basis of the approach developed in [3]. 


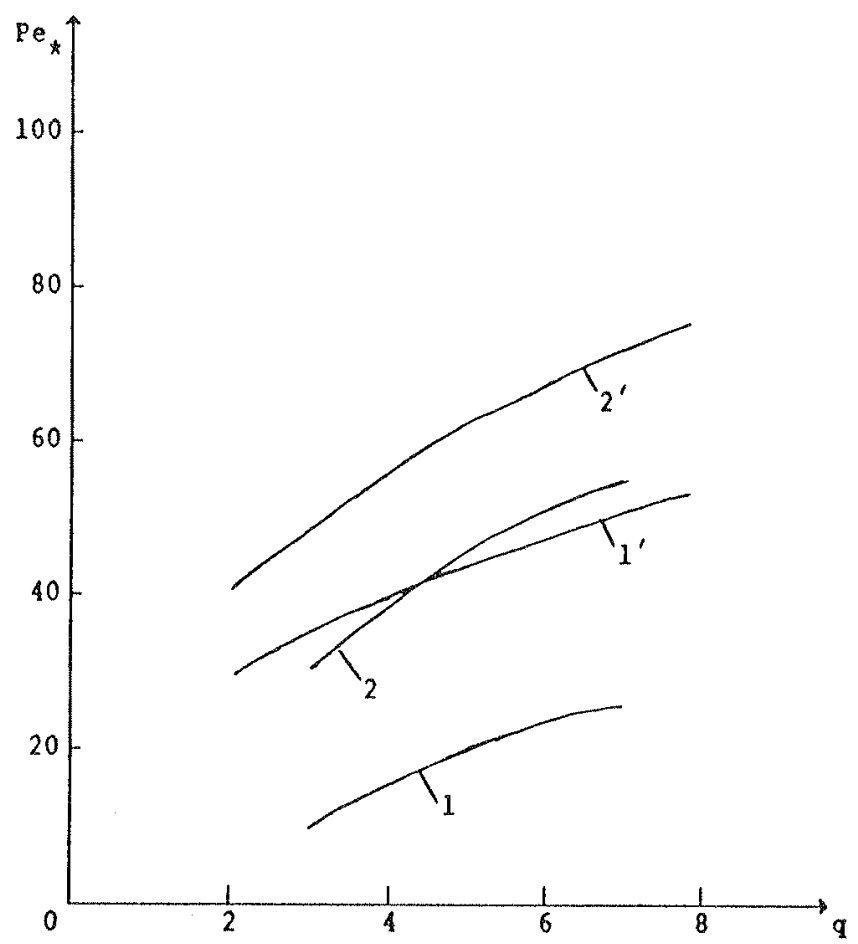

FIGURE 2. Dependencies of the critical Peclet number Pe* with the parameter $\mathrm{q}=\mathrm{Q} \cdot \mathrm{a}_{0} / \mathrm{C}_{\mathrm{v}} \cdot \mathrm{T}_{0} . \quad 1,2$ - numerical calculations; $1^{\prime}, 2^{\prime}$ - the calculation by the formulae (5), (6).

Using a one-dimensional approach let's consider a steady flame front propagating in a half-infinite channel with the cold side walls from the closed heat-impenetrable end. In the coordinate-system $\left(0^{\prime}, X^{\prime}\right)$, connected with the flame front this end moves in the negative direction with the velocity $U_{b}$. The end coordinate is $X_{0}=-U_{b} \cdot t$, the reaction products occupy the region $X^{\prime} \circ<X^{\prime}<0$, the fresh mixture $-0<X^{\prime}<\infty$; for $X^{\prime}=0$ is taken as the point where the reaction is finished; the chemical reaction zone is considered to be infinitely narrow. The heat balance per unit of the flame surface area may be written as:

$\rho_{b} \cdot U_{b} \cdot C_{p} \cdot\left(T_{a}-T_{b}\right)=\left.\lambda \cdot \frac{d T}{d X^{\prime}}\right|_{x^{\prime}=0}+\frac{U^{\prime} \alpha}{L} \cdot \int_{0}^{\infty}\left(T-T_{0}\right) d X^{\prime}$

and determines the fall in burning temperature in comparison with the adiabatic one; $\mathrm{T}_{\mathrm{a}}, \mathrm{T}_{\mathrm{b}}$ are the combustion temperatures under the adiabatic 
conditions and with concomitant heat losses; $\rho_{b}$ is the density of the combustion products ( $\rho_{b} U_{b}=\rho_{0} U_{n}$ - for the one-dimensional steady state flame); $\quad \alpha$ is the heat transfer coefficient, considered as constant; $v$ characterizes the symmetry of the problem: $v=2$ for the planar channel, $v=4$ for the round tube; $L$ is the width of the channel or the diameter of the tube.

The left hand term in the right side of (1) describes the heat losses from the burning zone to the cooling reaction products, the right hand one - the heat losses from the preheated flame zone. Substituting in the integral of the right part of (1), the Michelson's temperature distribution in the preheated flame zone, we have:

$\frac{v \cdot \alpha}{L} \cdot \int_{0}^{\infty}\left(T-T_{0}\right) d X^{\prime}=\frac{v \cdot \alpha \cdot\left(T_{b}-T_{0}\right) \cdot \lambda}{L \cdot \rho_{b} \cdot U_{b} \cdot C_{p}}$

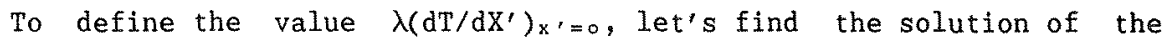
following problem:

$-\rho_{b} \cdot U_{b} \cdot C_{p} \cdot \frac{d T}{d X^{\prime}}=\frac{d}{d X^{\prime}} \lambda \frac{d T}{d X^{\prime}}-\frac{\nu \cdot \alpha \cdot\left(T_{o}-T\right)}{L}$

$\mathrm{X}^{\prime}=0: \quad \mathrm{T}=\mathrm{T}_{\mathrm{b}} ; \quad \mathrm{X}^{\prime}=\mathrm{X}^{\prime} \circ: \frac{\mathrm{dT}}{\mathrm{d} \mathrm{X}^{\prime}}=0$

which describes the temperature distribution in the reaction products.

Substitution of (2) and obtained solution of problem (3) in (1) gives:

$\frac{1}{\Delta}=\frac{\left(1+v \cdot \mathrm{Nu} / \mathrm{Pe}^{\prime}\right) \cdot v \cdot \mathrm{Nu} \cdot\left(1-\exp \left(\mathrm{k}_{1}-\mathrm{k}_{2}\right) \cdot \mathrm{X}_{0}\right)}{\mathrm{Pe}^{\prime 2} \cdot\left(1+v \cdot \mathrm{Nu} / \mathrm{Pe}^{\prime 2}\right)+v \cdot \mathrm{Nu} \cdot \exp \left(\mathrm{k}_{1}-\mathrm{k}_{2}\right) \mathrm{X}_{0}}+\frac{v \cdot \mathrm{Nu}}{\mathrm{Pe}^{\prime 2}}$

where $\Delta=\left(\mathrm{T}_{b}-\mathrm{T}_{0}\right) /\left(\mathrm{T}_{\mathrm{a}}-\mathrm{T}_{\mathrm{b}}\right), \mathrm{Pe}^{\prime}=\frac{\mathrm{L} \cdot \mathrm{U}_{\mathrm{b}} \cdot \rho_{\mathrm{b}} \cdot \mathrm{C}_{\mathrm{p}}}{\lambda}, \mathrm{Nu}=\frac{\alpha \cdot \mathrm{L}}{\lambda}$

$\mathrm{k}_{1}=\frac{\mathrm{Pe}^{\prime}}{\mathrm{L}} \cdot\left(1+\frac{\nu \cdot \mathrm{Nu}}{\mathrm{Pe}^{\prime 2}}\right), \mathrm{k}_{2}=-\frac{\nu \cdot \mathrm{Nu}}{\mathrm{L} \cdot \mathrm{Pe^{ \prime }}}$ 
The most interesting cases are: $X^{\prime} \circ \rightarrow 0$ - the flame is close to the end wall and $X^{\prime} \circ \rightarrow-\infty$ the flame moves away from the igniting end over long distance as a stationary one.

In the first $x^{\prime} \circ \rightarrow 0$, we may obtain:

$\mathrm{Pe}^{\prime}{ }_{1}=\sqrt{\mathrm{V} \cdot \mathrm{Nu} \cdot \Delta}$

In the second case corresponding to $X^{\prime} \circ \rightarrow-\infty$, from (4) it follows the expression:

$\mathrm{Pe}_{2}^{\prime}=\sqrt{2 \cdot \mathrm{V} \cdot \mathrm{Nu} \cdot \Delta}$

From (5) and (6) with an accuracy of $R_{0} \cdot T_{2} / \tilde{E}$ it follows from the connection between difference in temperature of the burning zone and the flame velocity is obtained in [3] $\mathrm{T}_{a}-\mathrm{T}_{b} \sim \mathrm{U}^{-2}$. Therefore it is possible to use the result obtained in [3]: at the limit $T_{a}-T_{*}=R_{0} \cdot T_{a}{ }^{2} / \tilde{E}$ and $\mathrm{U}_{*}=\mathrm{U}_{2} / \sqrt{\mathrm{e}}\left(\mathrm{U}_{\mathrm{a}}\right.$ - the flame velocity in the adiabatic conditions). Consequently for the limiting conditions the value $\Delta$ is known and is equal to $\Delta_{*}=\left(T_{a}-T_{0}\right) \cdot \tilde{E} / R_{0} \cdot T_{a}^{2}=q \cdot \tilde{E} / \gamma \cdot \theta_{a}$. The substitution $\Delta_{*}$ in (5) and (6) gives critical values of the Peclet numbers $-\mathrm{Pe}_{1}$ and $\mathrm{Pe}_{2}$.

Thus for $\mathrm{Pe}^{\prime} \geq \mathrm{Pe}^{\prime}{ }_{2}$ the flame is capable of propagation without quenching; for $\mathrm{Pe}_{1}<\mathrm{Pe}^{\prime}<\mathrm{Pe}^{\prime}{ }_{2}$ it quenches, after passing some distance, and for $\mathrm{Pe}^{\prime}<\mathrm{Pe}_{1}^{\prime}$ it quenches near the left wall.

In Fig.2 the calculation using formulas (5) and (6) gives $\nu=2, \Delta=\Delta$, $\mathrm{Nu}=3.75$. The qualitative coincidence of the variations of $\mathrm{Pe} *(q)$ allows the use of the obtained analytical formulae (5), (6) as rather good estimations.

For the study of the processes in the case, when the flame is able to propagate along all over the channel length, the series of calculations was carried out for different Reynolds numbers in the range $R e=50 \div 300$ by $q=5$.

In Fig. 3 are shown the time variations of the flame front coordinate $X_{f}$ (the point at the axis $x$, in which $a=0.5$ ), the average volume concentration $\langle a\rangle$ and the average volume temperature $\langle\theta\rangle$ for different Reynolds numbers. There are two distinctly marked parts of the curves $\langle a\rangle(t)$ and $X_{f}(t)$ - at the initial stage of the process where the curves are more steep, than later.

It can be explained by the fact that at first the flame spreads from the closed wall with the dimensionless velocity close to 1 . Then as the hot products of the reaction $\mathrm{cool}$, the heated gas expanding behind the flame front has less and less influence on the flame propagation speed and it becomes close to the normal flame speed $U=p_{b} \cdot U_{b} / p_{0}=0.22$ (the inclinations of the curves at the sloping parts $\simeq 0.2$ ). It's worth mentioning that in all the investigated range of the Reynolds numbers 50 


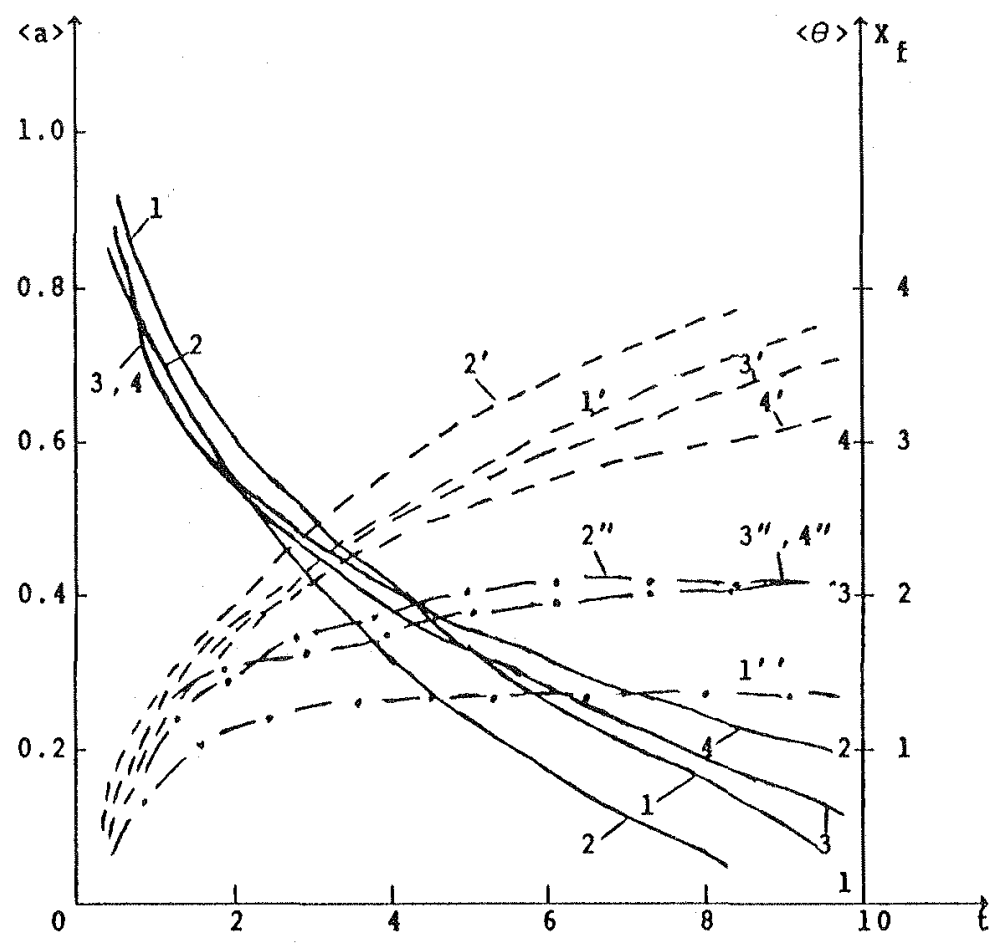

FIGURE 3. The time characteristics of the flame propagation process. $-\langle a\rangle(t) ; \quad-X_{f}(t) ; \quad-\langle\theta\rangle(t), 1,1^{\prime}, 1^{\prime \prime}-\operatorname{Re}=50$; $\overline{2,2^{\prime}}, 2^{\prime \prime}-\operatorname{Re}=100 \overline{0} \overline{3}, \overline{3}{ }^{\prime}, 3^{\prime}{ }^{\prime}-\operatorname{Re}=200 ;{ }^{-} 4,4^{\prime}, 4^{\prime} \prime-\operatorname{Re}=300$ 。

$\leq R e \leq 300$ the quasi-stationary thermal regimes exists, when the rate of the heat losses to the walls is balanced by the rate of chemical reaction heat release, this may be proved by the existence by Fig. 3 close to horizontal parts at the curves $\langle\theta\rangle(t)$.

\section{REFERENCES}

1. Makhviladze, G.M., Melikhov, V.I. and Melikhov, 0.I., "The Flame Propagation in a Close Volume", Fiz Goreniya $i$ Vzryva, 23: 3, 30-36, 1987. (In Russian).

2. Zeldovich, Ya.B., Barenblatt, G.I., Librovich, V.B., and Makhviladze, G.M. Mathematical Theory of Combustion and Explosion, pp. 1-583, Plenum Press, 1985. 
3. Zeldovich, Ya.B., "A Theory of the Limit of Slow Flame Propagation", Zh. Eksp. Teor. Fiz., 11: 1, 159-168, 1941. (In Russian).

4. Spalding, D.B., "A Theory of Inflammability Limits and Flame-Quenching", Proc. Roy. Soc. L., A240: 1220, 83-100, 1957.

5. Makhviladze, G.M., Shcherbak, S.B., "Numerical Method of Investigating Unsteady-State Spatial Movements of a Compressible Gas", Inq. Fiz. Zh., 38: 3, 528-537, 1980. (In Russian). 
